\title{
The association between continuity of care and the severity of diabetes-related complications
}

\author{
Jin-Yu He${ }^{1}$, Tao-Hsin Tung ${ }^{2}$, Jia-Ni Xue ${ }^{1}$, Pei-En Chen ${ }^{3}$, Ching-Wen Chien*1 \\ ${ }^{1}$ Institute for Hospital Management, Tsing Hua University, Shenzhen, China \\ ${ }^{2}$ Department of Medical Research and Education, Cheng Hsin General Hospital, Taipei, Taiwan, China \\ ${ }^{3}$ Association of Health Industry Management and Development, Taipei, Taiwan, China
}

Received: September 27, 2018

DOI: $10.5430 /$ jer.v5n1p20
Accepted: November 22, $2018 \quad$ Online Published: November 29, 2018

URL: https://doi.org/10.5430/jer.v5n1p20

\begin{abstract}
Purpose: To assess the association between continuity of care and severity of diabetes-related complications for geriatric diabetic patients.

Methods: A retrospective study using 2009-2013 Taiwan's National Health Insurance Research Database one million beneficiary version were conducted. Utilization data of 3,885 geriatric patients who newly had type 2 diabetes were studied. Five-year Continuity of Care Index (COCI) and the adapted Diabetes Complications Severity Index (aDCSI) of each subject were calculated. A Group-based trajectory modeling (GBTM) was used to distinguish patients with similar five-year COCI into different trajectories. A general estimating equation(GEE) was used to assess the association between COCI trajectories and aDCSI.

Results: The ratio of male to female in this study was 2:3. At the time of inclusion, the average age was 71.79 (4.65) years old. GBTM subjects can be divided into four different trajectories according to their COCI: low-level continuity of care trajectories, increasing continuity of care trajectories, decreasing continuity of care trajectory, and high-level continuity of care trajectory. After GEE analysis, the high continuity of care trajectories were associated with a significant decrease in aDCSI score.

Conclusion: The results of this study suggested better continuity of care was associated with less severity of diabetes-related complications for geriatric patients.
\end{abstract}

Key Words: Diabetes complications severity index, Continuity of care index, Geriatric diabetes, Group-based trajectory modeling

\section{INTRODUCTION}

The global prevalence of diabetes among adults over 18 years old has risen from $4.7 \%$ in 1980 to $8.5 \%$ in 2014 , World Health Organization(WHO) indicated that diabetes will be the seventh leading cause of death in $2030 .{ }^{[1]}$ Diabetes is a major cause of blindness, kidney failure, heart attacks, stroke and lower limb amputation which has seriously to affect patients' health and quality of life. ${ }^{[2]}$ Previous population- based study of type 2 diabetes in 1996-2003 showed that although incidence rate of type 2 diabetes was approximately stable after 1997; however the rate of occurring diabetesrelated complications, notably cardiovascular disease, continued to impose a substantial burden to many countries. ${ }^{[3]}$ Chan et al.'s (2009) study showed that early onset and long disease duration place Asian diabetes patients at high risk for cardiorenal complications. ${ }^{[4]}$ Corriere et al. (2013) anChina

*Correspondence: Ching-Wen Chien; Email: y13810002868@qq.com; Address: Institute for Hospital Management, Tsing Hua University, Shenzhen, 
alyzed disease characteristics of geriatric diabetes patients and found that they also suffered higher prevalence of peripheral vascular disease, heart disease, and stroke. ${ }^{[5]}$ Besides geriatric diabetic patients were more likely to experience geriatric syndromes such as falls, dementia, depression, and incontinence. ${ }^{[5]}$ Diabetes and its related complications often affect health and quality of life of geriatric diabetes patients and their families. This may be due to the current treatment of diabetes. By far the most common mode is "treatment rehabilitation -long-term follow-up". Some previous studies demonstrated that continuity of care may improve certain components of diabetes care such as control of glucose, blood pressure, serum cholesterol, and perhaps health-related quality of life. ${ }^{[6]}$ The association between continuity of care and outcomes, such as death or all-cause hospitalizations of clinical events, is uncertain. ${ }^{[7-9]}$ Therefore, what role of continuity of care plays in the context of improving severity of diabetes-related complications of geriatric diabetes patients is worth to notice. Therefore, we aimed to investigate the association between continuity of care of geriatric diabetes patients and severity of diabetes-related complications.

\section{MethodS}

\subsection{Database}

This study used 2009-2013 one million beneficiary version of the Taiwan National Health Insurance Research Database (NHIRD). Taiwan has implemented its National Health Insurance (NHI) program since March 1995. The NHI is a mandatory enrollment and government operated single-payer insurance system covering western medicine, Chinese traditional medicine and dental care in outpatient, emergency room and hospitalization services. The program currently has over 23 million citizens, representing around $99 \%$ of Taiwan's total population. For every five years since 2000, the Bureau of the NHI (BNHI) randomly selected one million NHI beneficiary to represent Taiwan's total population and form a one million beneficiary version of the NHIRD for research purposes after systematically encrypted all identification.

The NHIRD contains at most five diagnostic and procedural codes in ICD-9-CM (International Classification of Diseases, 9th and Clinical Modification revision), personal information and 16 charge items of each claim. All procedures were performed in accordance with the guidelines of our institutional ethics committee and adhered to the Declaration of Helsinki. Because the data source was in the public domain and anonymized, informed consent was not given. This study was exempted from review by the Institutional Review Board.

This study was a retrospective study of geriatric patients who Published by Sciedu Press were over 65 years old and newly diagnosed with type 2 diabetes (ICD-9-CM codes 250. $\times$ x, excluding $250 . \times 1$ or $250 . \times 3$ ) during 2009-2013. A patient defined as having diabetes, is diagnosed with diabetes by at least two doctors or medical institutions, both of which contain a diagnosis of diabetes and prescriptions. The date of the second diagnosis was used as the index date and then traced all utilization claim data related to type 2 diabetes for five consecutive years. Any subject who had fewer than two outpatient visits per year in two of the five follow-up years was also excluded from this study. The final study subjects consisted of 3,885 patients.

\subsection{Definition of variables}

Independent variables in this study include patients' age, gender (male/female), whether receiving any types of dialysis (yes/no), home care (yes/no) and catastrophic disease services (yes/no). A subject's continuity of care is measured by continuity of care index (COCI). ${ }^{[10-12]} \mathrm{COCI}$ is a widely used measure that incorporates the idea that personal continuity of care is affected by both the total number of diabetic care providers and the total number of ambulatory care visits. In this study, we used COCI derived from outpatient services in a period of time (one year), then calculated concentration at a single service provider concentration. ${ }^{[13]}$ The equation for calculating COCI is as follows:

$$
\mathrm{COCI}=\frac{\sum_{j=1}^{M} n_{j}^{2}-N}{N(N-1)}
$$

where $\mathrm{N}$ represents the total number of physician visits, $\mathrm{n}_{j}$ is the number of visits to the same physician, $\mathrm{j}$ represents a given physician, and $\mathrm{M}$ is the total number of physicians. ${ }^{[14]}$ We calculated COCI of each subject every 365 days (a year) for five consecutive years after his/her inclusion.

Dependent variable of this study is the adapted Diabetes Complications Severity Index (aDCSI). aDCSI that was developed by Chang et al. (2012) using a claim database of a US insurance company to demonstrate severity of the diabetes. ${ }^{[15,16]}$ aDCSI had been proved to be superior than the number of complications in explaining variation of medical expenditure due to complications. ${ }^{[16]}$ aDSCI is also more feasible to identify patient populations for subsequent disease management. ${ }^{[1]}$ Therefore, this study intends to use both diabetes complications (with/without) and aDCSI score as a measure of the severity of diabetes complications.

\subsection{Statistical analysis}

Statistical analyses were performed using SAS version 9.4. Continuous variables such as a subject's age were reported 
as means and standard deviation (SD) and categorical variables such as sex were expressed by their frequency na $\mathrm{d}$ percentile. A Group-Based Trajectory Modeling (GBTM) was used to divide subjects with similar COCI for five years into different trajectories. A generalized estimating equation (GEE) is used to assess the association between COCI trajectories and severity of diabetes complications and between different COCI trajectories. A $p$-value $<.05$ was considered statistically significant.

Table 1. The baseline characteristics of the study subjects ( $=3,885$ )

\begin{tabular}{ll}
\hline Variables & N(\%) or Mean (SD) \\
\hline Categorical Variables & \\
Sex & $1,560(40.15)$ \\
Male & $2,325(59.85)$ \\
Female & $27(0.69)$ \\
Receiving kidney dialysis service & $819(21.08)$ \\
Receiving nursing service (nursing home care) & $149(3.84)$ \\
Receiving major procedure (related operations) & 1,634 \\
Total of medical service provider & \\
Medical Department* & $14,015(31.43)$ \\
Endocrinology department & $11,475(25.74)$ \\
General medicine & $6,154(13.80)$ \\
Family medicine & $12,941(29.03)$ \\
Others & \\
Medical Service Type* & \\
Outpatient & $44,295(99.35)$ \\
Emergency & $61(0.14)$ \\
Hospitalization & $229(0.51)$ \\
Have diabetes-related complications & $1,224(31.51)$ \\
Continuous Variables & \\
Nue & $10.42(5.2)$ \\
Number of health institutions & $0.47(0.85)$ \\
\hline
\end{tabular}

*Total patient's visit number

\section{RESUlts}

Table 1 shows the descriptive statistics of the samples, shows the subjects' demographic characteristics, together with the received services and their medical department information. The male-female ratio was about $2: 3$, with a yearly similar distribution. The average age at the time of enrollment was 71.79 (4.65) years. For the related medical service, 0,69\% diabetes had dialysis services, $21.08 \%$ had nursing services, and $3.84 \%$ had overcome some kind of major procedure. In addition, Table 1 also indicates that diabetic patient visited
$1.42(0.7)$ medical institutions and the average number of effective outpatient visits in that year reached 10.42 (5.20). It could be observed that the total number of serious diabetic complications in the study subjects remained on a low score of aDCSI.

During the implementation of GBTM and the moment for characteristics distribution of COCI, it was subjectively selected the CNORM (Normal Censored) model. During the specific models screening process, the accepted BIC criterion was firstly taken into use, i.e. equation:

$$
2 \Delta \mathrm{BIC}=2\left(\mathrm{BIC}_{\text {complex }}-\mathrm{BIC}_{\text {null }}\right)>10
$$

Taking the variables BICnull and BICcomplex into consideration, it is possible to observe that the former belongs to simpler model while the latter to a more complicated one. Based on BIC value changes and its standard, referring to other 2 value also considering the clinical meaning to choose the model which is 4 groups.

Table 2. Evaluation results of the GBTM with study subjects $(n=3,885)$

\begin{tabular}{cccc}
\hline Group number & BIC & AIC & L \\
\hline 1 & $-11,531.62$ & $-11,519.09$ & $-11,515.09$ \\
2 & $-10,588.72$ & $-10,563.66$ & $-10,555.66$ \\
3 & $-10,519.28$ & $-10,481.69$ & $-10,469.69$ \\
4 & $-10,376.51$ & $-10,326.40$ & $-10,310.40$ \\
\hline
\end{tabular}

The Table 2 indicates the BIC, AIC and L values of each regression equation for group 1 to 4 , BIC value is the lowest in 4 groups, the Figure 1 is the corresponding track graph of 4 groups model [low-level $(\mathrm{n}=90,323.2 \%)$, increasing $(\mathrm{n}=$ $102,226.3 \%)$, decreasing $(\mathrm{n}=66,817.2 \%)$, high-level $(\mathrm{n}=$ 129, 233.3\%)]. There were significant differences between the four groups in the continuity of care measured by COCI scores, presented as follow: Mean low-level group's showed fluctuations annually, however, the overall level was the lowest among the four groups. Referring to the largest in the elevated group; the average value of COCI in the elevated group increased yearly, reaching almost 1.00 at the end of the five year follow up. The lowering group, on the contrary, was completely the opposite, and the average value of COCI decreased yearly. In the following years, the continuity kept decreasing, and; the high-level group remained stable at 1.00 in the tracking year (see Table 3).

It could also be observed how their outpatient visits continuity starts to develop a changing tendency and we following this statement, the obtained data highlights the following results: the first group's outpatient visit continuity remained 
low (Low-level continuity group), the second group's the outpatient visits continuity showed an annually increasing rate (increasing continuity group), the third group's outpatient visits continuity on the other hand showed a clear annually dropping rate (decreasing continuity group), and the fourth group's outpatient visit continuity remained as high-level continuity group.

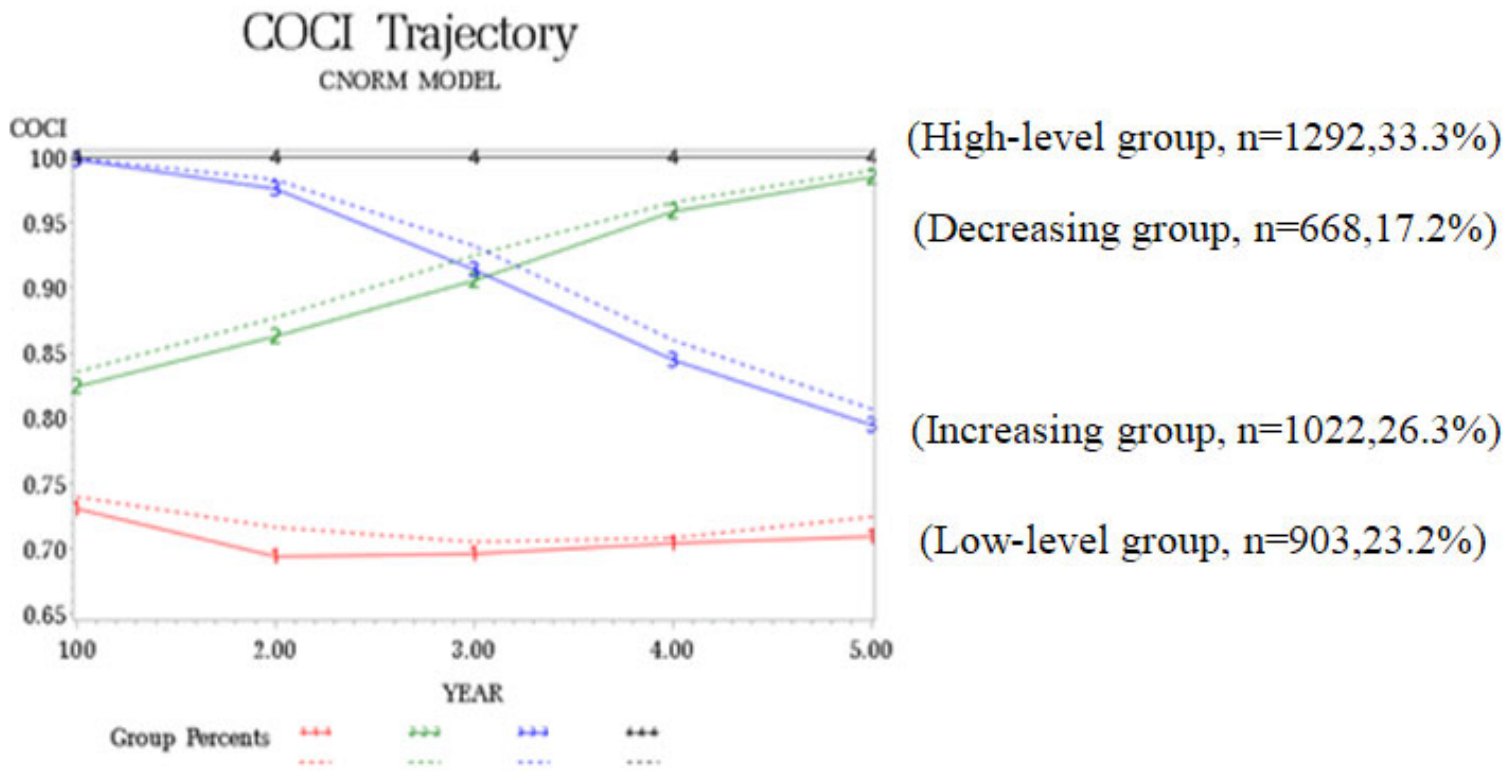

Figure 1. The trajectory of Continuity of Care $(n=3,885)$

Table 3. The COCI value of different groups in different years $(n=3,885)$

\begin{tabular}{lllllllll}
\hline $\begin{array}{l}\text { Continuity level } \\
\text { of care }\end{array}$ & $\begin{array}{l}\text { Study } \\
\text { Subjects }\end{array}$ & Statistics & $\begin{array}{l}\text { The year be } \\
\text { chosen }\end{array}$ & Year1 & Year2 & Year3 & Year4 & Year5 \\
\hline Low-level & \multirow{2}{*}{903} & Mean & 0.7539 & 0.7181 & 0.6707 & 0.6844 & 0.6801 & 0.6878 \\
& & SD & {$[0.2651]$} & {$[0.2553]$} & {$[0.2453]$} & {$[0.2543]$} & {$[0.2353]$} & {$[0.2416]$} \\
Increasing & \multirow{2}{*}{1,022} & Mean & 0.8577 & 0.8034 & 0.8414 & 0.8720 & 0.9715 & 0.9943 \\
& & SD & {$[0.2209]$} & {$[0.2195]$} & {$[0.2089]$} & {$[0.1926]$} & {$[0.0968]$} & {$[0.0340]$} \\
Decreasing & \multirow{2}{*}{668} & Mean & 0.9108 & 1.0000 & 0.9917 & 0.9271 & 0.7964 & 0.7440 \\
& \multirow{2}{*}{} & SD & {$[0.1823]$} & {$[0.0000]$} & {$[0.0426]$} & {$[0.1588]$} & {$[0.2205]$} & {$[0.2261]$} \\
High-level & \multirow{2}{*}{1,292} & Mean & 0.9273 & 1.0000 & 1.0000 & 1.0000 & 1.0000 & 1.0000 \\
& & SD & {$[0.1680]$} & {$[0.0000]$} & {$[0.0000]$} & {$[0.0000]$} & {$[0.0000]$} & {$[0.0000]$} \\
\hline
\end{tabular}

Based on the results of GEE model for diabetic complications, Table 4 indicates that the grouping variables coefficients were all negative and significant $(p<.05)$. After a comparison between the low-level continuity group, the increasing continuity group, the decreasing continuity group and the high-level continuity group it can be observed a significant reduction on diabetic complications incidences. Table 5 shows that from the GEE model fitting results, the coefficients of the three grouped dummy variables are all negative and significant at the significance level of 0.05. After comparison among the low continuity group, the population of the continuous continuity group, the reduced continuity group, and the high continuity group, a significant decrease in aDCSI score in subsequent years was shown. In addition, it is conjectured that the improvement in care continuity is the most pronounced for the reduction of aDCSI scores compared to the group where care continuity has been low.

\section{Discussion}

\subsection{Strengths and weaknesses of this study}

This study examined the association between continuity of medical care and severity of diabetes-related complications. 
We selected the indicator COCI as the initial measure of care continuity, which has strongest explanatory power and is independent of the number of visits. The group trajectory model was used to fit the trend of annual COCI values for each sample individual for five consecutive years. Through this study, it is found that there is a certain regularity in the continuity of the treatment of diabetic patients, and there is a relatively fixed trend in a period of time. In addition, in the subsequent empirical research, the grouping variable generated by the group trajectory model has always had a strong explanatory significance, which proves to some extent the rationality of using the model in this study.

Table 4. Results of GEE model on aDCSI $(n=3,885)$

\begin{tabular}{|c|c|c|c|c|c|}
\hline Variable & B & Standard Error & OR & $95 \% \mathrm{CI}$ & $P$ Value \\
\hline \multicolumn{6}{|l|}{ Model 1} \\
\hline \multicolumn{6}{|l|}{ Continuity level of care } \\
\hline Low-level continuity group & --- & --- & 1.00 & --- & --- \\
\hline Increasing continuity group & -0.30 & 0.08 & 0.74 & $(0.64,0.86)$ & .0001 \\
\hline Decreasing continuity group & -0.38 & 0.08 & 0.68 & $(0.58,0.81)$ & $<.0001$ \\
\hline High-level continuity group & -0.47 & 0.07 & 0.62 & $(0.54,0.72)$ & $<.0001$ \\
\hline \multicolumn{6}{|l|}{ Year effect } \\
\hline Year 1 & --- & --- & 1.00 & & - \\
\hline Year 2 & 0.13 & 0.04 & 1.14 & $(1.06,1.22)$ & .0002 \\
\hline Year 3 & 0.22 & 0.04 & 1.24 & $(1.15,1.34)$ & $<.0001$ \\
\hline Year 4 & 0.26 & 0.04 & 1.29 & $(1.19,1.41)$ & $<.0001$ \\
\hline Year 5 & 0.27 & 0.05 & 1.30 & $(1.19,1.43)$ & $<.0001$ \\
\hline
\end{tabular}

*Adjustment for confounding factors: Age, Sex, Dialysis, nursing services (nursing home care), Catastrophic disease.

Table 5. Results of GEE model for adjusted diabetes complication severity index among elderly type 2 diabetes $(\mathrm{n}=3,885)$

\begin{tabular}{|c|c|c|c|c|c|}
\hline Variable & B & Standard Error & $95 \% \mathrm{CI}$ & & $P$ Value \\
\hline \multicolumn{6}{|l|}{ Continuity level of care } \\
\hline Low-level continuity group & --- & --- & --- & --- & --- \\
\hline Increasing continuity group & -0.20 & 0.06 & -0.31 & -0.09 & .0004 \\
\hline Decreasing continuity group & -0.18 & 0.07 & -0.32 & -0.05 & .007 \\
\hline High-level continuity group & -0.17 & 0.05 & -0.28 & -0.07 & .0012 \\
\hline \multicolumn{6}{|l|}{ Year effect } \\
\hline Year 1 & --- & --- & --- & --- & --- \\
\hline Year 2 & -0.04 & 0.03 & -0.10 & 0.01 & .1432 \\
\hline Year 3 & -0.10 & 0.03 & -0.16 & -0.04 & .0011 \\
\hline Year 4 & -0.14 & 0.03 & -0.21 & -0.08 & $<.0001$ \\
\hline Year 5 & -0.18 & 0.04 & -0.25 & -0.11 & $<.0001$ \\
\hline
\end{tabular}

*Adjustment for confounding factors: a DCSI(at the beginning), Nursing service (nursing home care).

It should be noted that this study has several limitations. older. This sub-group may have differences in the concept of First, this study depended on claims data with limited scope. medical care, medical service provision, and accounting for Therefore, this study did not include unavailable variables treatment expenses from other age groups. At the same time, (such as occupation, socioeconomic status and accession for some special characters of Taiwan's health care system, to the health service) in the analyses. In addition, the sub- it may make difficult to generalize the results of this study to jects selected for analysis in this study were aged 65 and other health care systems. Future study requires the studies 
to other health care systems and cultures.

\subsection{Results in relation to other comparable studies}

We identified six other research articles which theme include "continuity of care" and "diabetes" since 2013 (see Table 6). Most of them take mortality and hospitalization rates as the indicator of severity of the diabetes. They show that high continuity of care can prevent the progression of renal disease. ${ }^{[17]}$ Greater continuity of care was associated with reduced risk of subsequent death and hospitalization. ${ }^{[10,18-20]}$
Only in Lustman et al.'s research, when adjusting for background characteristics the difference between in OR for hospital admissions became non-significant 0.92 (0.84-1.01). ${ }^{\text {[21] }}$ Although none of them takes aDCSI as an index to reflect the outcomes of care, the most conclusions are consistent, It was suggested that greater continuity of care are related to better outcome (lower motility, fewer complications, fewer hospitalization admission). It is showed that good continuity is associated with better indicator results which contribute to less severity of diabetes-related complications.

Table 6. The continuity of care among elderly type 2 diabetes in various populations

\begin{tabular}{|c|c|c|c|c|c|c|c|}
\hline $\begin{array}{l}\text { First } \\
\text { author }\end{array}$ & $\begin{array}{l}\text { Study } \\
\text { year }\end{array}$ & $\begin{array}{l}\text { Subjects } \\
\text { Number }\end{array}$ & Place & COCI & Complication & Hospitalization & Reference \\
\hline \multirow{5}{*}{$\begin{array}{l}\text { Chang et } \\
\text { al. }\end{array}$} & \multirow{5}{*}{2018} & \multirow{5}{*}{26,063} & \multirow{5}{*}{ Taiwan, China } & & ESRD & & \multirow{5}{*}{ [17] } \\
\hline & & & & & & & \\
\hline & & & & Low & $190(46.12 \%)$ & $\mathrm{aHR}=1.72$ & \\
\hline & & & & Intermediate & 130 (31.55\%) & $\mathrm{aHR}=1.15$ & \\
\hline & & & & High & 92 (22.33\%) & $\mathrm{aHR}=1.00$ & \\
\hline Pan et al. & 2017 & 396,838 & Taiwan, China & high (> 50\%) & $\begin{array}{l}\text { Mortality } \\
\text { HR: } 0.47\end{array}$ & $\longrightarrow$ & [18] \\
\hline $\begin{array}{l}\text { Lustman et } \\
\text { al. }\end{array}$ & 2016 & 23,294 & Clalit & high & $\begin{array}{l}\text { Mortality } \\
\text { OR=0.59 }\end{array}$ & $\begin{array}{l}\mathrm{aOR}=0.92 \\
\text { No significant }\end{array}$ & [21] \\
\hline Weir et al. & 2016 & 285,231 & US & $\mathrm{UPC} \geqslant 75 \%$ & $\begin{array}{l}\text { death or } \\
\text { hospitalization }\end{array}$ & $\begin{array}{l}\mathrm{aOR}=0.72 \\
(13.5 \% / 7.2 \%)\end{array}$ & [19] \\
\hline Cho et al. & 2015 & $1,100,000$ & Korea & UPC $<75 \%$ & NA & OR:2.44 & [24] \\
\hline Hong et al. & 2013 & 68,469 & Korea & COCI $<0.4$ & NA & $\mathrm{OR}=1.37$ & [10] \\
\hline
\end{tabular}

UPC: Usual Provider of Continuity; COCI: Continuity of Care Index

\subsection{Meaning of the study and possible explanations}

Diabetes and its complications usually need a long-term treatment process. Most patients suffering from diabetes and its complications visit the doctor regularly to get the treatment and medicine. In the actual situation, each patient chooses his/her attending physician within a certain range due to the limitations of physical condition, personal preference, income level, geographical location and other subjective and objective factors. And in many cases, patients may not be able to get continuous treatment due to improper time, limited economic conditions or shortage of medical resources, etc. The health condition deteriorates due to poor management of existing diseases and even causes other complications. This study explore the relationship between continuity of medical care and severity of diabetes-related complications. High-level continuity means that the communication and interaction between diabetic patients and their doctors will be more frequent than other patients, which can help the doctor get more information about the patient, for instance, the patient's various physical indications, changes in disease development, etc. All above can be better understood and definitely help doctors to develop more scientific treatment programs without unnecessary examinations and medications. From the patient's point of view, High-level continuity because the doctor visits the same doctor for a long time, the patient will have a better understanding of the doctor's diagnosis and treatment habits and work attitudes, thus gradually establishing trust in the doctor, and will be more willing to cooperate with the doctor during the follow-up treatment. All of these will inevitably help to produce better diagnosis and treatment results.

\subsection{The clinical suggestion}

Community family physician model is one of the effective means to promote continuity of care. Since the United Kingdom in 1948 regarded family physicians as the "gatekeeper" 
of residents' health management and take it into the National Health Service (NHS), there are many countries in the world that have implemented family physician systems. There may be some differences between different countries, but the family doctor's knowledge structure is the same. It involves prevention, health care, medical care, rehabilitation and other aspects, and it should provide patients with comprehensive, continuous and personalized medical services. Previous studies did by Zhang Fei et al. ${ }^{[22]}$ and Liang Huanqi et al. ${ }^{[23]}$ also proved that the family doctor service contributes to diabetes by better physiological indicators control and improving their health management.

In additions, with the breakthroughs in technologies for sensor networks and the Internet of Things, it has brought a lot of convenience for doctors to collect real-time data of patients by wearable devices and communication between doctors and patients is less difficult than before. Future research should pay more attention to how to make good use of rapidly developing network and Technology and combine

\section{REFERENCES}

[1] Mathers CD, Loncar D. Projections of global mortality and burden of disease from 2002 to 2030. PLoS Med. 2006; 3: e442. PMid:17132052. https://doi.org/10.1371/journal.pmed.0 030442

[2] World Health Organization- Diabetes Key facts. WHO 2017.

[3] Ringborg A, Lindgren P, Martinell M, et al. Prevalence and incidence of Type 2 diabetes and its complications 1996-2003-estimates from a Swedish population-based study. Diabetic Med. 2008; 25: 1178-1186. PMid:19046196. https://doi.org/10.1111/j.1464-5491.20 $08.02541 . x$

[4] Chan JC, Malik V, Jia W, et al. Diabetes in Asia epidemiology, risk factors, and pathophysiology. JAMA. 2009; 20: 2129-2140. PMid:19470990. https://doi.org/10.1001/jama.2009.726

[5] Corriere M, Rooparinesingh N, Kalyani RR. Epidemiology of diabetes and diabetes complications in the elderly: an emerging public health burden. Curr Diab Rep. 2013; 6: 1-14. https ://doi .org/ 10.1007/s11892-013-0425-5

[6] Hussey PS, Schneider EC, Rudin RS, et al. Continuity and the costs of care for chronic disease. JAMA Intern Med. 2014; 174: 742-748. PMid:24638880. https://doi.org/10.1001/jamainternmed. 2014.245

[7] Overland J, Yue DK, Mira M. Continuity of care in diabetes: to whom does it matter? Diabetes Res Clin Pract. 2001; 52: 55-61. https : //doi .org/10.1016/S0168-8227(00)00240-0

[8] Woodard LD, Landrum CR, Urech TH, et al. Impact of clinical complexity on the quality of diabetes care. Am J Manag Care. 2012; 18: 508-514. PMid:23009301.

[9] Knight JC, Dowden JJ, Worrall GJ, et al. Does higher continuity of family physician care reduce hospitalizations in elderly people with diabetes? Popul Health Manag. 2009; 12: 81-86. PMid:19361251. https://doi.org/10.1089/pop. 2008.0020 it with family doctor to improve the care continuity, reduce the incidence of future complications of diabetic patients and prevent the deterioration of existing diseases.

\section{Conslusion}

In conclusion, this study revealed that better continuity of care was associated with less severity of diabetes-related complications seen from lower probabilities of the occurrence of diabetes-related complications and lower aDCSI score. Given these findings, we recommend that health authorities should promote continuity of care by educating patients about benefices of better continuity of medical care and provide more accesses to get continuous medical care for elderly type 2 diabetes.

\section{AUTHORS' CONTRIBUTION}

The first two authors contributed equally to this article.

\section{CONFlicts OF InTEREST Disclosure}

The authors declare that they have no competing interests.

[10] Hong JS, Kang HC. Continuity of ambulatory care and health outcomes in adult patients with type 2 diabetes in Korea. Health Policy. 2013; 109: 158-165. PMid:23093021. https://doi.org/10.101 6/j.healthpol.2012.09.009

[11] Gill JM, Mainous AG, Diamond JJ, et al. Impact of provider continuity on quality of care for persons with diabetes mellitus. Ann Fam Med. 2003; 1: 162-170. PMid:15043378. https ://doi .org/10.1 370/afm. 22

[12] Hong JS, Kang HC, Kim J. Continuity of care for elderly patients with diabetes mellitus, hypertension, asthma, and chronic obstructive pulmonary disease in Korea. J Korean Med Sci. 2010; 25: 1259-1271.

[13] Reid R, Haggerty J, McKendry R. Defusing the confusion: concepts and measures of continuity of health care. Ottawa, Ontario: Canadian Health Services Research Foundation; 2002.

[14] Bice TW, Boxerman SB. A quantitative measure of continuity of care. Med care. 1977; 15: 347-349. https ://doi.org/10.1097/0000 5650-197704000-00010

[15] Chang HY, Weiner JP, Richards TM, et al. Predicting costs with diabetes complications severity index in claims data. Am J Manag Care. 2012; 18: 213-219. PMid:22554010.

[16] Chang HY, Weiner JP, Richards TM, et al. Validating the adapted diabetes complications severity index in claims data. Am J Manag Care. 2012; 18: 721-726. PMid:23198714.

[17] Chang PY, Chien LN, Bai CH, et al. Continuity of care with physicians and risk of subsequent hospitalization and end-stage renal disease in newly diagnosed type 2 diabetes mellitus patients. Ther Clin Risk Manag. 2018; 14: 511-521. PMid:29559787. https: //doi.org/10.2147/TCRM.S150638

[18] Pan CC, Kung PT, Chiu LT, et al. Patients with diabetes in pay-forperformance programs have better physician continuity of care and survival. Am J Manag Care. 2017; 23: E57-E66. PMid:28245660.

[19] Weir DL, McAlister FA, Majumdar SR, et al. The interplay between continuity of care, multimorbidity, and adverse events in pa- 
tients with diabetes. Med Care. 2016; 54: 386-393. PMid:26807539. https://doi.org/10.1097/MLR.0000000000000493

[20] Cho KH, Nam CM, Choi Y, et al. Impact of continuity of care on preventable hospitalization of patients with type 2 diabetes: a nationwide Korean cohort study. Int J Qual Health Care. 2016; 28: 478-485. PMid:27272406. https://doi.org/10.1093/intqhc/mzw050

[21] Lustman A, Comaneshter D, Vinker S. Interpersonal continuity of care and type two diabetes. Prim Care Diabetes. 2016; 10: 165-170. PMid:26530317. https://doi.org/10.1016/j.pcd.2015.10. 001
[22] Zhang F. Effect analysis of family doctor service team in the community health education for diabetes. China Medicine and Pharmacy. 2014; 14: 138-139.

[23] Liang HL, Wang YL, Luo ZZ. Efficacy of Family Doctor Service in Type 2 Diabetes Management, Acta Medicinae Universitatis Scientiae et Technologiae Huazhong. 2014; 43: 471-475.

[24] Cho KH, Lee SG, Jun B, et al. Effects of continuity of care on hospital admission in patients with type 2 diabetes: Analysis of nationwide insurance data. BMC Health Serv Res. 2015; 15: 107. PMid:25879858. https://doi.org/10.1186/s12913-015-0745-z 